\title{
Correction
}

\section{Correction to: Free Path Lengths in Quasicrystals}

\author{
Jens Marklof ${ }^{1}$, Andreas Strömbergsson ${ }^{2}$ \\ ${ }^{1}$ School of Mathematics, University of Bristol, Bristol BS8 1TW, UK. \\ E-mail: j.marklof@bristol.ac.uk \\ 2 Department of Mathematics, Uppsala University, Box 480, 75106 Uppsala, Sweden. \\ E-mail: astrombe@math.uu.se
}

Published online: 2 January 2020 - ( $\odot$ Springer-Verlag GmbH Germany, part of Springer Nature 2019

\section{Correction to: Commun. Math. Phys. 330, 723-755 (2014) https://doi.org/10.1007/s00220-014-2011-3}

In order for Theorem 5.1 to be correct, the definition of the Siegel transform, (5.1), should read as follows:

$$
\hat{f}(\Gamma h)=\sum_{\substack{\boldsymbol{m} \in \mathbb{Z}^{n} h g \\ \pi(\boldsymbol{m}) \neq \mathbf{0}}} f(\boldsymbol{m}) .
$$

The proof of Theorem 5.1 has to be corrected by replacing each occurrence of " $\backslash\{\boldsymbol{0}\}$ " (except the one in p. 747, line 4) by " $\backslash \pi^{-1}(\{\mathbf{0}\})$ ". Also in the proof of Corollary 5.2, each occurrence of " $\backslash\{\boldsymbol{0}\}$ " should be replaced by " $\backslash \pi^{-1}(\{\boldsymbol{0}\})$ ", and on p. 747, line -3, “0 $\notin \mathbb{Z}^{n} h g+\boldsymbol{z}$ ” should be replaced by " $\pi^{-1}(\mathbf{0}) \cap\left(\mathbb{Z}^{n} h g+\boldsymbol{z}\right)=\emptyset ”$.

Publisher's Note Springer Nature remains neutral with regard to jurisdictional claims in published maps and institutional affiliations.

Communicated by L. Erdös 